\title{
Design and fabrication of advanced EUV diffractive elements
}

\author{
Patrick P. Naulleau, J. Alexander Liddle, Farhad Salmassi, Erik H. Anderson, \\ and Eric M. Gullikson \\ Center for X-Ray Optics, Lawrence Berkeley National Laboratory, Berkeley, CA 94720
}

\begin{abstract}
As extreme ultraviolet (EUV) lithography approaches commercial reality, the development of EUV-compatible diffractive structures becomes increasingly important. Such devices are relevant to many aspects of EUV technology including interferometry, illumination, and spectral filtering. Moreover, the current scarcity of high power EUV sources makes the optical efficiency of these diffractive structures a paramount concern. This fact has led to a strong interest in phase-enhanced diffractive structures. Here we describe recent advancements made in the fabrication of such devices.
\end{abstract}

Keywords: extreme ultraviolet lithography, diffraction, grating, diffusers, holographic optical element

\section{INTRODUCTION}

Extreme ultraviolet (EUV) lithography ${ }^{1}$ remains the top candidate for the technology to be used for the 32-nm generation of nano-electronics expected to enter volume production in 2009. As EUV lithography approaches commercial reality, the development of EUV-compatible diffractive structures becomes increasingly important. Diffractive structures have and are expected to continue to play crucial roles in many aspects of EUV technology. Currently, the most prevalent role for diffractive structures in EUV technology is EUV wavefront metrology. The phase-shifting point-diffraction interferometer (PS/PDI), ${ }^{2,3}$ for example, relies on accurate grating beamsplitters and sub-50-nm spatial-filtering pinholes to measure optical systems with numerical apertures (NA) exceeding 0.25 . ${ }^{4}$ The EUV implementation of the lateral shearing interferometer (LSI) ${ }^{5,6}$ also relies intimately on grating beamsplitters including crossed gratings. ${ }^{7}$ Presently these gratings are typically transmission amplitude structures, however, significant benefit could be achieved in terms of throughput if transmission phase gratings were available.

Another promising area for diffractive EUV structures is in the implementation of high-efficiency spectral-purity filters, or monochromators. Although EUV systems are based on reflective optics, and are thus in principle relatively insensitive to chromatic errors, illumination bandwidth control is still required for EUV lithography. For example, appreciable amounts of UV power combined with resist sensitivity to this wavelength band would decrease printed image contrast. Also, appreciable amounts of IR power would place unacceptable thermal loads on the projection optics. Because of source power limitations in the EUV band of interest, it is crucial, however, that the EUV efficiency of the bandwidth control be maximized. Moreover, because IR rejection is a desired goal of the system, thermal robustness of the bandwidth control system is also of utmost importance. These requirements make blazed-phasegrating-based monochromators a very attractive option compared to membrane transmission filters. ${ }^{8}$

Another promising area for diffractive EUV devices is in structured illumination systems. ${ }^{9}$ Often times EUV sources intrinsically provide significantly lower divergence then might be required for imaging conditions. EUV-compatible random scatter plates and holographic or diffractive optical elements could play important roles in structured illumination systems potentially providing both coherence and illumination uniformity control. Again, the desire for high efficiency leads to a strong preference for phase-enhanced devices.

\section{TRANSMISSION PHASE STRUCTURES}

As described above, EUV wavefront interferometers could benefit significantly from the availability of transmission phase gratings. Not only could throughput gains be achieved for both the PS/PDI and the LSI, but the practically achievable accuracy of the PS/PDI might also be enhanced. ${ }^{10}$ While it is difficult to find pure phase-shifting materials at EUV wavelengths, there are various candidate materials that have attractive absorption to phase-shifting ratio properties. ${ }^{11}$ 
One of the best available materials for phase shifting at EUV wavelengths is Molybdenum (Mo). At a wavelength of $13.4 \mathrm{~nm}$, Mo has a Delta (the decrement from unity of the real part of the complex index of refraction) of $7.73 \times 10-2$ and a Beta (the imaginary part of the index of refraction) of $6.23 \times 10-3$. From these values, we find the 1/e attenuation length of Mo to be $171 \mathrm{~nm}$ and the $\pi$-phase-shift length to be $86.5 \mathrm{~nm}$. For a $\pi$-phase-shift thickness the transmission intensity at a wavelength of $13.4 \mathrm{~nm}$ is approximately $60 \%$.

Mo has recently been used to fabricate phase-enhanced gratings with promising results. ${ }^{10}$ The efficacy of the present process has been limited by the quality of the wet Mo etch chemistry used, however, improved dry etch processes are being developed. To date, for a $40.7 \%$ duty cycle grating (mostly open) first order to zero-order diffraction efficiency ratios of up to 2.4 have been achieved, whereas a ratio approaching 4 is possible. Significantly higher ratios are also feasible simply by increasing the duty cycle towards $56 \%$ where the peak ratio occurs. We note that peak specular suppression is not at $50 \%$ as one might typically expect from a phase grating due to residual absorption of EUV in Mo.

Another potentially interesting application for Mo transmission devices is an EUV diffuser, an EUV equivalent to etched or ground glass as often used at visible wavelengths. As described below, however, the efficiency of such a device is severely limited by the residual, albeit relatively small, absorption of EUV by Mo.

For the following illustrative analysis, we consider a conventional statistically Gaussian diffuser ${ }^{12}$ where the minimum thickness is set to zero. In the case of an ideal phase diffuser, the desired spatial bandwidth can be equally-well achieved by varying either the minimum feature size on the diffuser and/or the modulation-depth of the diffuser. For a Mo diffuser, however, the inherent attenuation leads to a trade-off between modulation-depth and efficiency. For this reason, it is beneficial to use spatial feature size as the primary means of obtaining the desired diffusion bandwidth. It is also important to note that the dc-suppression (suppression of the specular component that leads to a coherent background) requirement leads to a lower limit on the acceptable modulation-depth.

Table 1 shows the low-angle scatter to specular transmission ratio (diffuser efficacy) and total efficiency for Gaussian Mo diffusers of varying modulation-depth. The diffusers are arbitrarily chosen to have a bandwidth in the 0.01 to 0.02 NA range. The modulation-depth is defined as the standard deviation of the thickness profile in $\pi$-phase-shift lengths. For example, a Mo Gaussian diffuser with a modulation-depth of 1 has a thickness standard deviation of $86.5 \mathrm{~nm}$. The low-angle scatter to specular transmission ratio is defined as shown in Fig. 1, which is a line-out along the $\mathrm{x}$ axis of the calculated far-field scatter pattern for the modulation-depth equal 1 case. Figure 1 shows the time-averaged angular scatter profile assuming the diffuser to be moving at a rate of more than 1000 correlation lengths over the integration time.

The tabulated diffuser properties are based on far-field diffraction calculations. The time averaging, which is required when considering properties related to coherence, is simulated by calculating the intensity diffraction patterns for many (more than 1000) independent realizations of the diffuser and averaging the results. We note that the total power efficiency shown in Table 1 is calculated ignoring substrate effects. In practice, the diffuser must be patterned onto a membrane that will further attenuate the light, further diminishing the throughput. If, for example, the diffuser is patterned onto a $100-\mathrm{nm}$ thick $\mathrm{Si}_{3} \mathrm{~N}_{4}$ membrane, the actual efficiency will be $\sim 43 \%$ of the numbers shown in Table 1 .

\begin{tabular}{|c|c|c|}
\hline Modulation depth & efficacy & total power efficiency (\%) \\
\hline 1.0 & 0.52 & 13.8 \\
\hline 1.1 & 0.82 & 11.5 \\
\hline 1.2 & 0.96 & 9.7 \\
\hline 1.4 & 0.98 & 6.8 \\
\hline 4.0 & 1.00 & 0.9 \\
\hline
\end{tabular}

Table 1. Diffuser properties as a function of modulation depth. 
From Table 1 we see that a modulation depth of greater than 1.2 is required in order to achieve an efficacy greater than 0.96 for the $\sim 0.02$ NA bandwidth Molybdenum diffusers considered here. We also see that exceeding this modulation depth causes the efficiency to quickly drop below $10 \%$, making it likely impractical to achieve an efficacy of unity. We note, however, that requiring an efficacy of unity may be overly restrictive: when used as a decoherentizer for an imaging system, the more relevant property to consider is the ratio of the incoherent-image, or partially-coherent-image, forming light to coherent-image forming light. The specular component can be viewed as simply overlaying the desired partial-coherence image with a full-coherence image. In this case, the ratio of interest is the total energy contributing to the imaging over the specular energy contributing to the imaging. For the first entry in Table 1, where the efficacy is 0.52 , we see this new ratio to be 7,769 to 1 under the assumption that scattering from the diffuser up to an NA of 0.025 contributes to the image (the acceptance NA of a $4 \times$-reduction 0.1-NA system). The incoherent image is more than 7,000 times stronger than the coherent overlay. This apparent tremendous gain is due to this new measure taking into consideration the two-dimensional nature of the diffuser. The efficacy definition in Fig. 1 ignores the effect of integration over two-dimensional scattering angle.

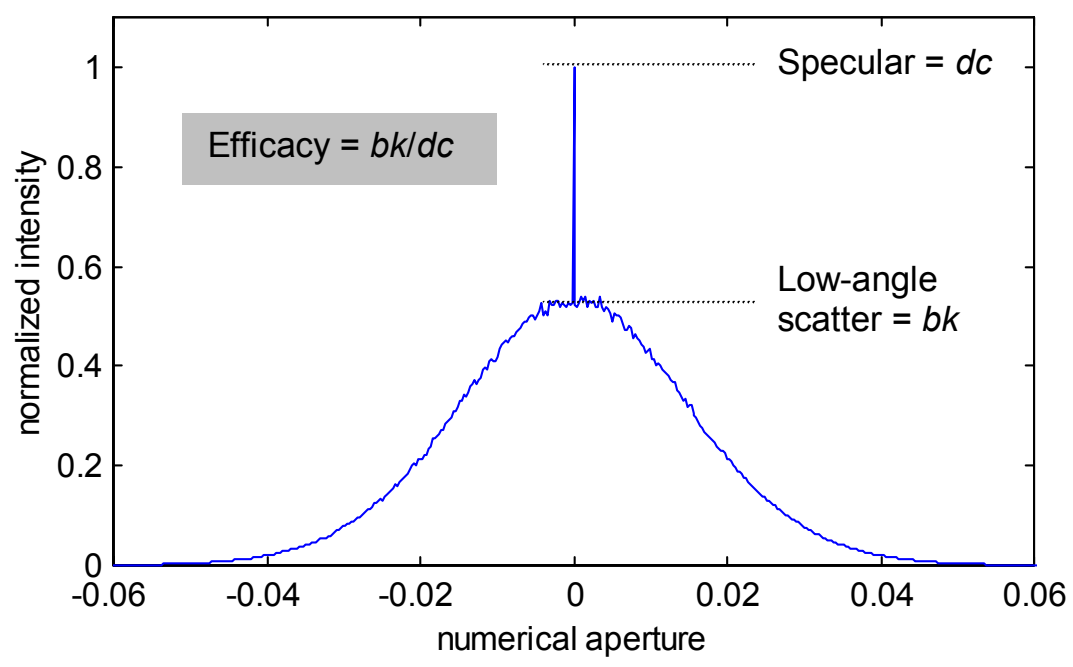

Fig. 1. Definition of diffuser efficacy.

The claim that the incoherent image is more than 7,000 times stronger than the coherent image is based on the assumption that both the coherent and incoherent object-plane illumination areas are equal in size. This limiting case is only achieved when the diffuser is very close to the object plane or re-imaged to the object plane (Critical Illumination). If the diffuser is Fourier transformed to the object plane (Khöler Illumination) the coherent illumination will be concentrated. ${ }^{13}$ In this case the coherent image corruption may be more noticeable, however, it will be limited in area.

\section{REFLECTION BLAZED PHASE GRATINGS}

Spiller ${ }^{14}$ was the first to suggest the use of multilayer reflection coatings ${ }^{15,16}$ to improve the efficiency of gratings in the soft X-ray and EUV wavelength ranges. Since that time, considerable effort has been directed to the implementation of this idea at various wavelengths and angles of incidence. ${ }^{17-26}$ Of particular interest has been the implementation of blazed gratings due to potentially high diffraction efficincies, ${ }^{19-23}$ however, in practice, square-wave gratings had been found to deliver higher first-order efficiency. ${ }^{24-26}$ This is due to superior substrate control that had been achieved in the past with square-wave gratings as compared to that achieved with blazed gratings.

More recently, however, gray-scale e-beam lithography techniques have been developed enabling the fabrication of extremely high efficiency near-normal incidence blazed phase grating operating in the EUV regime. ${ }^{27,28}$ Noting that an 8 -step blazed phase reflective grating operating at a wavelength of $13.4 \mathrm{~nm}$ and and angle of incidence of $5^{\circ}$ would ideally have a peak-to-valley height of $5.88 \mathrm{~nm}$ and an individual step height of $0.841 \mathrm{~nm}$, the fabrication task is quite 
challenging. Fabrication of the desired grating profile is achieved directly in resist through a gray-scale electron-beam exposure process. With high resolution and attainable roughness of lower than $1 \mathrm{~nm}$ rms, hydrogen silsesquioxane (HSQ), ${ }^{29}$ a spin on glass made by Dow Corning, has proven ideal for this application. Another benefit of this material is that it is extremely stable after development, serving as a thermally stable permanent base for the requisite multilayer overcoat.

In the recently developed HSQ grating fabrication process, ${ }^{28}$ the grating profile is patterned into a approximately 80 -nm thick layer of resist spun onto a silicon wafer. For the exposure step, a high-resolution electron-beam lithography tool $^{30,31}$ operating at $100 \mathrm{keV}$, is utilized. The desired grating is represented as a data set wherein the desired grating heights as a function of position are encoded as different relative doses to be delivered to the resist. The prescribed doses include compensation for resist nonlinearity ${ }^{32}$ and localized back-scatter effects. The base dose is approximately $300 \mu \mathrm{C} / \mathrm{cm}^{2}$. After exposure, the HSQ is developed in $0.26 \mathrm{~N}$ TMAH photoresist developer, Shipley LDD26W for 75 seconds.

The next step is to overcoat the grating with a reflective Mo/Si multilayer. Two separate coating processes have been used: magnetron deposition ${ }^{33}$ more faithfully preserves the underlying topography, whereas ion-beam deposition ${ }^{34}$ can be used to provide enhanced smoothing of the residual roughness in the resist. In practice, we have found the two methods to yield nearly identical results, ${ }^{28}$ with the smoothing gains achieved by the ion-beam process being offset by losses in topographic fidelity. We note that this conclusion is expected to depend somewhat on the pitch of the grating. Although parity between the two methods was found at a pitch of $1 \mu \mathrm{m}$, one would expect the magnetron process to be preferable as the period is reduced and the ion-beam process to be preferable as the period is increased.

Using the above-described processes, $1-\mu \mathrm{m}$ pitch EUV gratings with absolute diffraction efficiencies as high as $41 \%$ into the first diffracted order have been demonstrated. ${ }^{28}$ Given that typical multilayer reflectivities in the EUV range are on the order of $65 \%$, these results demonstrate the high quality of the profiles achievable with the e-beam fabrication method even after multilayer coating.

Noting that a grating can be viewed as a null hologram, the methods described above can readily be extended to the fabrication of computer-generated holograms (CGH). Using the blazed grating as the carrier is best suited to the fabrication of phase only holograms. Alternatively, the same fabrication method can be used to generate a strictly binary carrier more readily enabling the fabrication of phase-amplitude holograms. The amplitude can be encoded into the carrier by modulating the duty cycle and hence the diffraction efficiency. This has advantages over patterning of a second absorbing layer because overlay requirements are eliminated. The drawback of going to a binary carrier instead of the blazed carrier is that the efficiency is reduced. An ideal binary phase carrier would support a theoretical efficiency of only $40 \%$ instead of $100 \%$ of the multilayer reflectivity. Nevertheless, this represents a significant improvement over what could be achieved using a binary amplitude carrier where the efficiency would be limited to $10 \%$ of the multilayer reflectivity.

A significant limitation of the overcoated CGH process, however, is the multilayer smoothing effect. In practice one will be limited in resolution by the smoothing instead of the lithography. Noting that the multilayer smoothing effect can support at best a resolution of 100-nm for a typically EUV multilayer and that e-beam tools have been shown to support fabricated structures exceeding $25-\mathrm{nm}$ resolution, ${ }^{31}$ using the overcoating process described here can represent a factor of 10 reduction in attainable resolution. This can be significant for CGH applications because of the large spatial bandwidth requirements of carrier-frequency holography. ${ }^{35,36}$

\section{REFLECTION EUV DIFFUSERS}

The low efficiency of the transmission EUV diffuser described above would render it impractical in many applications. Methods similar to those used to fabricate EUV blazed phase gratings, however, are also applicable to the fabrication of EUV reflection diffusers.

A reflection diffuser, imparting the phase modulation by virtue of geometric path-length differences instead index of refraction changes, requires much less topography than does the transmission Mo diffuser. Moreover, the fact that the device provides nearly pure phase modulation means that the modulation depth, as defined above, can safely approach unity further reducing the required topography. Nevertheless, fabricating a true Gaussian diffuser ${ }^{12}$ could, in principle, require arbitrarily large topography because there remains a finite probability of any height. To avoid this issue, the 
diffuser topography can be safely limited to a height range of $+/-3 \sigma$. Targeting a modulation depth of 1.1 , a wavelength of $13.4 \mathrm{~nm}$, and an angle of incidence of $10^{\circ}$, would lead to a required peak-to-valley topography range of $22.6 \mathrm{~nm}$, approximately 4 times larger than the topography required of the HSQ grating described in section 3.

The $22.6 \mathrm{~nm}$ topography requirement applies after multilayer coating. In practice, the topography patterned into the HSQ must be even larger and will depend strongly on the coating process used as well as the target correlation length for the diffuser. Taking into consideration magnetron-sputtered multilayer smoothing effects combined with a target spatial correlation length of approximately $100 \mathrm{~nm}$, we find the actual required topography to exceed $30 \mathrm{~nm}$. As the target correlation length is reduced or the multilayer smoothing is increased, the required pre-coating topography will also increase.

Reoptimizing the HSQ process described in section 3 for this larger topography, the diffuser profile is patterned into approximately a $360-\mathrm{nm}$ thick layer of resist. The exposure step is again performed using an electron-beam lithography tool operating at $100 \mathrm{keV}$, with the desired Gaussian topography being represented as relative dose as described above. The base dose is approximately $100 \mu \mathrm{C} / \mathrm{cm}^{2}$ and the development time is 120 seconds. The HSQ surface is then overcoated with a magnetron sputtered Mo/Si multilayer to provide the EUV reflectivity. In the case of the 100-nmcorrelation-length diffuser, magnetron coating has been found to be preferable over ion-beam deposition due to the lower smoothing of the patterned roughness.

A 100-nm correlation length diffuser fabricated using this process has been characterized using the high accuracy EUV reflectometer at the calibration and standards bend-magnet beamline 6.3.2 at the Advanced Light Source located at Lawrence Berkeley National Laboratory. ${ }^{37}$ The measurement was performed with a spectral resolution, $\lambda / \Delta \lambda$, of approximately 1400 . The diffuser was characterized by placing a detector at the re-imaging plane of the exit slit of the beamline monochromator and scanning the detector through the diffraction pattern. The illuminating beam was $200-\mu \mathrm{m}$ wide guaranteeing it to not spill over the $0.6 \times 1.8 \mathrm{~mm}$ diffuser area. This in turn assures an accurate measurement of the diffuser scatter characteristics.

Figure 2 shows a line scan through the center of the far-field diffraction pattern. The overlain smoothed curve is a fit to the data showing that the diffraction pattern is well approximated out to approximately $4^{\circ}$ by a Lorentzian-squared function. No significant specular peak is observed. The measured total efficiency of the diffuser is approximately $21 \%$ and the efficiency within the target 100 -nm correlation length is approximately $12.2 \%$. Assuming an efficacy of 0.96 , as defined in section 2, a transmission Mo diffuser fabricated onto a 100-nm-thick silicon nitride membrane would have

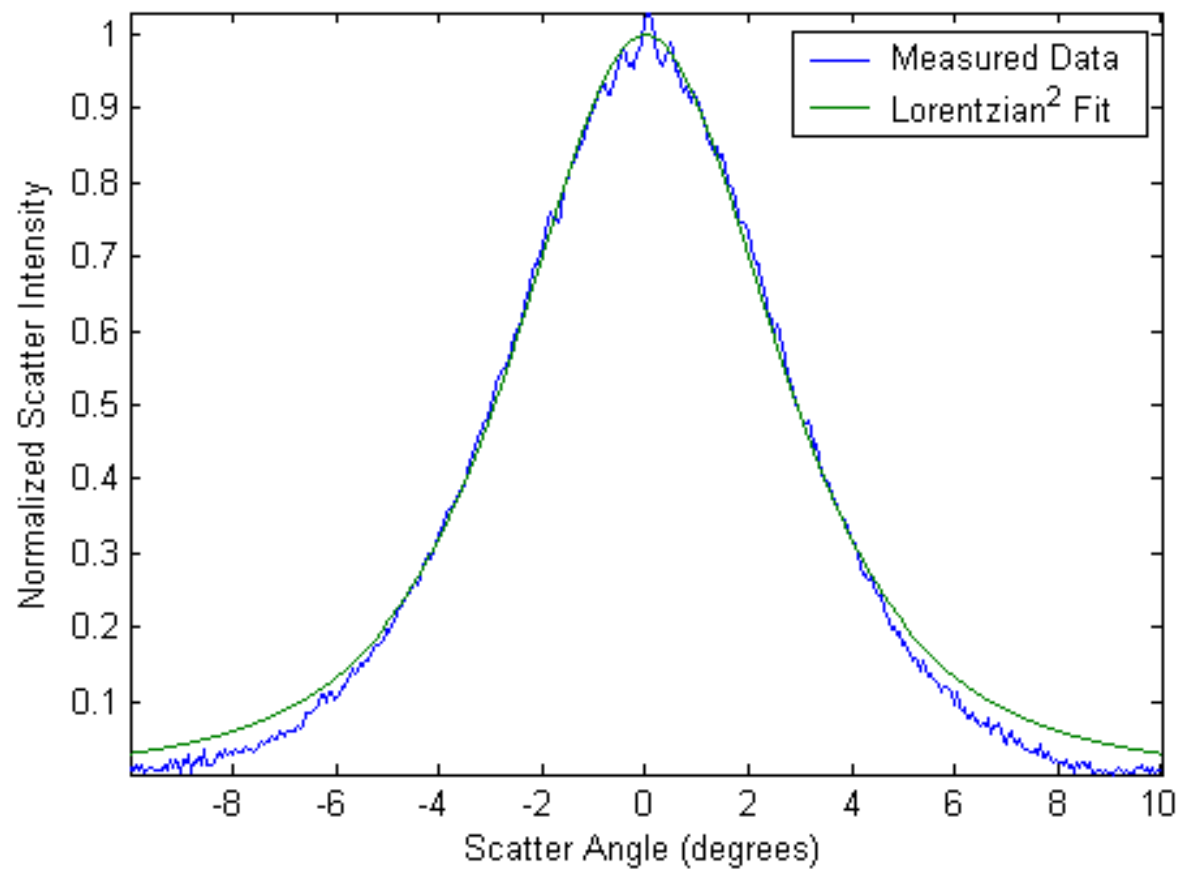

Fig. 2. EUV-measured scatter data for a reflective $\sim 100$-nm correlation length EUV diffuser fabricated using gray-scale e-beam lithography in HSQ. 
an ideal total efficiency of approximately 4\%, 5 times lower then the demonstrated efficiency of the HSQ reflection diffuser.

Although powerful in terms of its flexibility, the HSQ diffuser fabrication process is extremely slow due to the exposure process. It takes approximately 6 hours to expose a $1-\mathrm{mm}^{2}$ area of diffuser. This method is evidently not feasible for large area diffusers. To address this issue we have also studied the fabrication of diffuser substrates where the desired roughness is engineered through a sputtering process.

Process studies in this area have included a variety of materials, however, our best results have been achieved in chrome. In this method, a magnetron-sputtering chamber is used to sputter the chrome. By adjusting the energetics of the process and target distance parameters, one can exercise some control over the roughness of the sputtered surface. This method has the capability to produce nearly arbitrarily large diffusers. In our particular sputter chamber, surfaces up to $100-\mathrm{mm}$ in diameter can be coated, producing nearly $8,000 \mathrm{~mm}^{2}$ of diffuser area in less than an hour. Moreover, larger capability magnetron tools are readily available. Using the e-beam technique, a diffuser of equivalent area would take approximately 6 years of exposure time.

Figure 3 shows the EUV-measured scatter profile for a recently fabricated large-area chrome diffuser. The smooth curve is again a fit to the data and in this case a Gaussian-squared function is found to provide a good fit up to an angle of $10^{\circ}$. As with the HSQ diffuser, no significant specular peak is observed. The measured total efficiency of the diffuser is approximately $7.6 \%$ and the efficiency within the HSQ diffuser target 100-nm correlation length is approximately $2.5 \%$. A significant drawback of this method is that it is very difficult to control correlation length of the resulting diffuser resulting in much less control over the diffusion characteristics and often leading to a negative impact on the efficiency compared to an optimized HSQ diffuser.

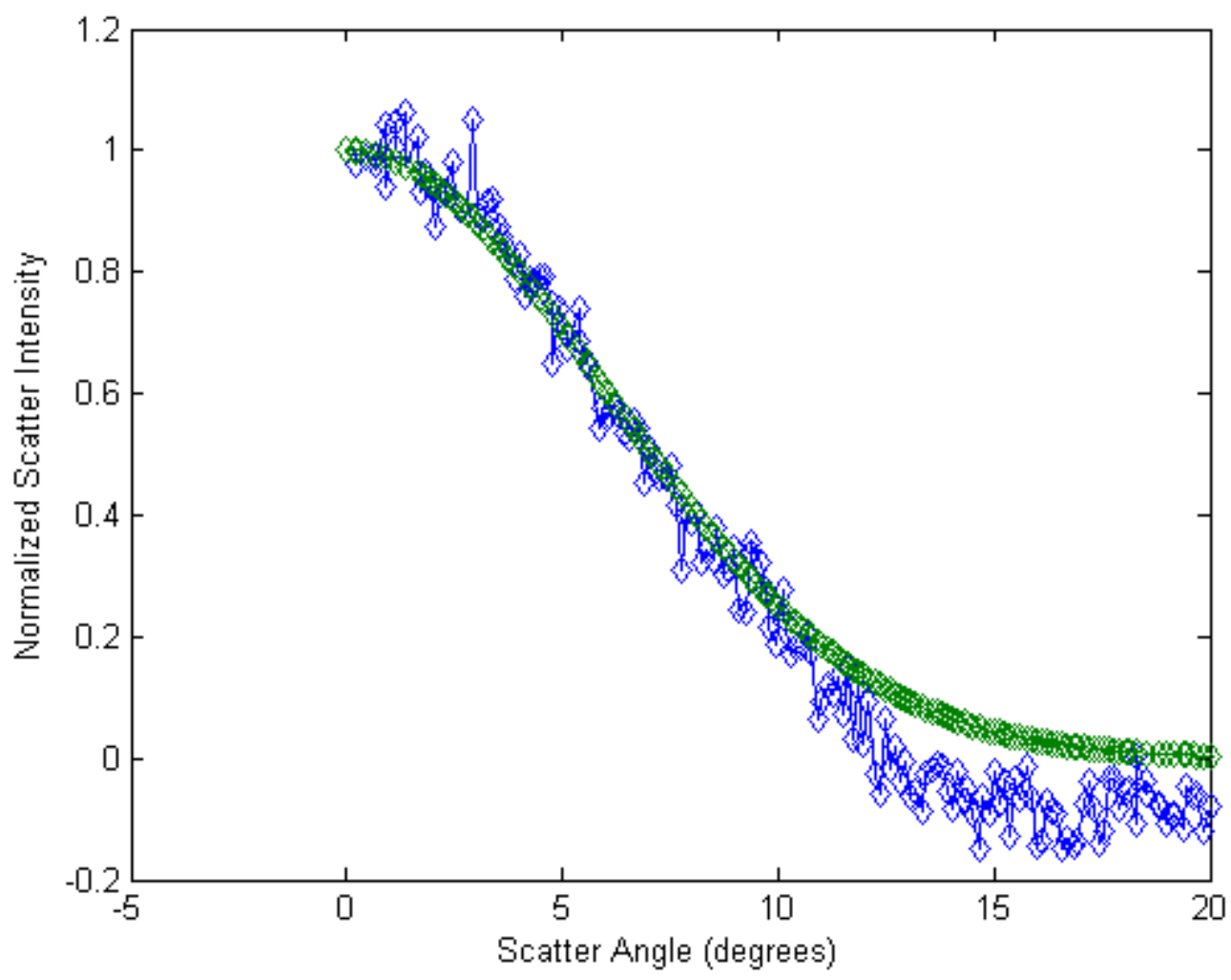

Fig. 3. EUV-measured scatter data for a reflective sputtered-chrome diffuser. 
Finally we note that it is also feasible to fabricate an EUV diffuser using the CGH techniques described in section 3. In addition to the constraints described in section 3, a diffuser implemented as a CGH will force the diffuse light to be placed onto a spatial carrier.

\section{SUMMARY}

The development of EUV lithography has spurred interest in diffractive EUV elements. The efficiency of these elements is of utmost concern driving the development of phase-enhanced structures. To address these needs we have developed a variety of new fabrication techniques supporting both transmission and reflection phase elements. Here we have summarized results demonstrating transmission phase gratings potentially important for metrology techniques, high-efficiency blazed phase reflection gratings with great promise for use in spectral-purity filters, and reflection diffusers for use in structured illumination systems.

\section{ACKNOWLEDGEMENTS}

The authors are greatly indebted to Bruce Harteneck and Eugene Veklerov for expert programming and fabrication support, and to the entire CXRO staff for enabling this research. This research was supported by the Extreme Ultraviolet Limited Liability Company and the Director, Office of Science, Office of Basic Energy Science, of the US Department of Energy under contract No. DE-AC03-76SF00098.

\section{REFERENCES}

1. R. Stulen and D. Sweeney, "Extreme ultraviolet lithography," IEEE J. Quantum Electron. 35, 694-699 (1999).

2. H. Medecki, E. Tejnil, K. A. Goldberg, and J. Bokor, Opt. Lett., 21, 1526-1528 (1996).

3. K. A. Goldberg, Ph.D. dissertation (University of California, Berkeley, 1997).

4. K.A. Goldberg, P. Naulleau, P. Denham, S. Rekawa, K. Jackson, E. Anderson, J. Liddle and J. Bokor, "AtWavelength Interferometry of the 0.3 NA MET Optic," J. Vac. Sci. \& Technol. B, to be published, (2004).

5. J. E. Bjorkholm, A. A. MacDowell, O. R. Wood II, Z. Tan, B. LaFontaine, and D. M. Tennant, "Phase-measuring interferometry using extreme ultraviolet radiation,” J. Vac. Sci. \& Technol. B, 13, 2919-2922 (1995).

6. A. K. Ray-Chaudhuri, W. Ng, F. Cerrina, Z. Tan, J. Bjorkholm, D. Tennant, and S. J. Spector, "Alignment of a multilayer-coated imaging system using extreme ultraviolet Foucault and Ronchi interferometric testing," J. Vac. Sci. Technol. B, 13, 3089-3093 (1995).

7. P. Naulleau, K. A. Goldberg, and J. Bokor, "EUV carrier-frequency shearing interferometry of a lithographic fourmirror optical system,” J. Vac. Sci. \& Technol. B 18, 2939-2943 (2000).

8. P. Naulleau, W. Sweatt, and D. Tichenor, "Theoretical efficiency analysis of a condenser-embedded grating-based spectral purity filter for EUV lithography," Opt. Comm. 214, 31-38 (2002).

9. P. Naulleau, K. Goldberg, E. Anderson, P. Batson, P. Denham, S. Rekawa, and J. Bokor, "Adding static printing capabilities to the EUV phase-shifting point diffraction interferometer," Proceedings of the SPIE Vol. 4343, 639-645 (2001).

10. P. Naulleau, C. Cho, E. Gullikson, and J. Bokor, “Transmission phase gratings for EUV interferometry," J. Synch. Rad. 7, 405-410 (2000).

11. B. L. Henke, E. M. Gullikson, and J. C. Davis. "X-ray interactions: photoabsorption, scattering, transmission, and reflection at E=50-30000 eV, Z=1-92," Atomic Data and Nuclear Data Tables, 54, 181-342 (1993).

12. J. W. Goodman, Statistical Optics, John Wiley \& Sons, New York, 1986, Chap. 8, 361-464.

13. J. W. Goodman, Statistical Optics, John Wiley \& Sons, New York, 1986, Chap. 7, 286-360.

14. E. Spiller, "Evaporated multilayer dispersion elements for soft X-rays," AIP Conf. Proc. 75, 124-130, (1981).

15. E. Spiller, "Reflective multilayer coatings for the far UV region," Appl. Opt. 15, 2333-2338, (1976). 
16. J. H Underwood and T. W. Barbee, Jr., "Layered synthetic microstructures as Bragg diffractors for X rays and extreme ultraviolet: theory and predicted performance," Appl. Opt. 20, 3027-3034, (1981).

17. R. A. M. Keski-Kuha, "Layered synthetic microstructure technology considerations for the extreme ultraviolet," Appl. Opt. 23, 3534-3537, (1984).

18. W. Jark, "Enhancement of diffraction grating efficiencies in the soft X-ray region by a multilayer coating," Opt. Comm. 60, 201-205, (1986).

19. J. C. Rife, W. R. Hunter, T. W. Barbee, Jr., and R. G. Cruddace, "Multilayer-coated blazed grating performance in the soft x-ray region," Appl. Opt. 28, 2984-2986, (1989).

20. R. A. M. Keski-Kuha, R. J. Thomas, J, S. Gum, and C. E. Condor, "Performance of multilayer coated diffraction gratings in the EUV," Appl. Opt. 31, 4529-4531, (1990).

21. J. F. Seely, M. P. Kowalski, W. R. Hunter, J. C. Rife, T. W. Barbee, Jr., G. E. Holland, C. N. Boyer, and C. M. Brown, "On-blaze operation of a Mo/Si multilayer-coated concave diffraction grating in the 136-142- $\AA$ wavelength region and near normal incidence," Appl. Opt. 32, 4890-4897, (1993).

22. J. H. Underwood, C. K. Malek, E. M. Gullikson, and M. Krumrey "Multilayer-coated echelle gratings for soft $x$ rays and extreme ultraviolet," Rev. Sci. Instrum. 66, 2147-2150, (1995).

23. M. P. Kowalski, T. W. Barbee, Jr., R. G. Cruddace, J. F. Seely, J. C. Rife, and W. R. Hunter, "Efficiency and longterm stability of a multilayer-coated, ion-etched blazed holographic grating in the 125-133- $\AA$ wavelength region," Appl. Opt. 31, 7338-7346, (1995).

24. M. P. Kowalski, R. G. Cruddace, J. F. Seely, J. C. Rife, K. F. Heidemann, U. Heinzmann, U. Kleineberg, K. Osterried, D. Menke, and W. R. Hunter, "Efficiency of a multilayer-coated, ion-etched laminar holographic grating in the 14.5-16.0-nm wavelength region," Opt. Lett. 22, 834-836, (1997).

25. J. F. Seely, M. P. Kowalski, R. G. Cruddace, K. F. Heidemann, U. Heinzmann, U. Kleineberg, K. Osteried, D. Menke, J. C. Rife, and W. R. Hunter, "Multilayer-coated laminar grating with 16\% normal-incidence efficiency in the 150-Å wavelength region," Appl. Opt. 36, 8206-8213 (1997).

26. C. Montcalm, S. Bajt, and J. F. Seely, "MoRu-Be multilayer-coated grating with $10.4 \%$ normal-incidence efficiency near the 11.4-nm wavelength,” Opt. Lett. 26, 125-127, (2001).

27. P. Naulleau, E. Anderson, E. Gullikson, and J. Bokor "Fabrication of high-efficiency multilayer-coated binary blazed gratings in the EUV regime," Opt. Comm. 200, 27-34 (2001).

28. P. Naulleau, J. A. Liddle, E. H. Anderson, E. M. Gullikson, P. Mirkarimi, F. Salmassi, and E. Spiller, "Fabrication of high-efficiency multilayer-coated gratings for the EUV regime using e-beam patterned substrates," Opt. Comm., to be published (2003).

29. F. van Delft, J. Weterings, A. van Langen-Suurling, H. Romijn, "Hydrogen silsesquioxane/novolak bilayer resist for high aspect ratio nanoscale electron-beam lithography," J. Vac. Sci. Technol. B 18, 3419-3423 (2000).

30. E. H. Anderson, V. Boegli, and L. P. Muray, "Electron beam lithography digital pattern generator and electronics for generalized curvlinear structures,” J. Vac. Sci. Technol. B 13, 2529-2534 (1995).

31. E. H. Anderson, D. L. Olynick, B. Harteneck, E. Veklerov, Gregory Denbeaux, W. Chao, A. Lucero, L. Johnson, and D. Attwood, "Nanofabrication and diffractive optics for high-resolution x-ray applications," J.Vac. Sci. Technol. B 18, 2970-2975 (2000).

32. J. A. Liddle, F. Salmassi, P. Naulleau and E. Gullikson, "Nanoscale topography control for the fabrication of advanced diffractive optics," J. Vac. Sci. Technol. B, to be published (2003).

33. D. Stearns, R. Rosen, and S. Vernon, "Fabrication of high-reflectance Mo-Si multilayer mirrors by planarmagnetron sputtering," J. Vac. Sci. Technol. A 9, 2662-2669 (1991).

34. E. Spiller, S. Baker, P. Mirkarimi, V. Sperry, E. Gullikson, and D. Stearns, "High Performance Mo/Si Multilayer Coatings for EUV Lithography using Ion Beam deposition," Appl. Opt. 42, 4049-4068, (2003).

35. E. N. Leith and J. Upatnieks, "Reconstructed wavefronts and communication theory," J. Opt. Soc. Am., 52, 11231130 (1962). 
36. E. N. Leith and J. Upatnieks, "Wavefronts reconstruction with diffused illumination and three-dimensional objects," J. Opt. Soc. Am., 54, 1295-1302 (1964).

37. E. M. Gullikson, S. Mrowka, B. Kaufmann, "Recent Developments in EUV Reflectometry at the Advanced Light Source," Proc. SPIE Vol. 4343, 363-373 (2001). 